\title{
Comparative study between early active and passive rehabilitation protocols following two-strand flexor tendon repair: can two-strand flexor tendon repair withstands early active rehabilitation?

\author{
Hala M. Abdel Sabour ${ }^{\mathrm{a}}$, Amir Labib ${ }^{\mathrm{b}}$, Ahmed Abel Sallam ${ }^{\mathrm{b}}$, Mohey Elbanna ${ }^{\mathrm{c}}$
}

Departments of aPhysical Medicine, Rehabilitation and Rheumatology, ${ }^{\text {bPlastic }}$ Surgery, 'General Surgery, Faculty of Medicine, Ain Shams University, Cairo, Egypt

Correspondence to Hala M. Abdel Sabour MD, Department of Physical Medicine, Rheumatology and Rehabilitation, Faculty of Medicine, Ain Shams University, 56 Ramsis Street, Abbasseya, Cairo 11566, Egypt. Tel/fax: 02 26830729;

e-mail: halams@medu.asu.edu.eg

Received 31 May 2018

Accepted 21 June 2018

Egyptian Rheumatology \& Rehabilitation 2018, 45:125-132

\begin{abstract}
Background
Restoration of full range of motion of digits as well as prevention of joint contracture following flexor tendon repair is a challenge. There is lack of solid evidence regarding the most suitable rehabilitation protocol following flexor tendon repair. This is owing to the limited number of studies comparing different rehabilitation protocols. Moreover, the present studies advocate a specific technique with no comparative group. Even the few controlled studies conducted vary in methods of repair and rehabilitation, and outcome assessment. To our knowledge, the only randomized controlled trial comparing early passive rehabilitation with early active rehabilitation is the one done by Trumble and colleagues in 2010, which was done on four-strand repaired tendon. These authors concluded that active rehabilitation program had better range of motion with less flexion contractures and greater satisfaction scores than those subjected to passive rehabilitation protocol.

Aim

This conclusion stimulated us to study the effect of early active mobilization versus early passive mobilization following two-strand repair.

Patient and methods

We conducted our study for 12 weeks comparing early active mobilization protocol 'place and hold' with early passive mobilization 'modified Kleinert' after standard two-strand modified Kessler repair in different hand zones.

Results and conclusion

We concluded that early active mobilization had better tendon gliding and excursion even with the two-strand repair as active motion will decrease adhesion formation, with significant difference compared with the passive group. Moreover, there was no significant difference in the rupture rate and significant difference for combined tendon lag and flexion deformity owing to the tenodesis mobilization between both the groups.
\end{abstract}

\section{Keywords:}

flexion deformity, flexor tendon repair, rehabilitation, tendon lag, two-strand suture, modified Kleinert

Egypt Rheumatol Rehabil 45:125-132

(C) 2018 Egyptian Society for Rheumatology and Rehabilitation $1110-161 \mathrm{X}$

\section{Introduction}

Despite the great advance in understanding flexor tendon anatomy, physiology, biomechanical mechanism of excursion and gliding, healing process and postoperative rehabilitation, still there is a significant rate of failure [1].

There are multiple points to keep in mind regarding tendon function postoperatively. There is always a problematic dilemma to balance between decreased rupture rate and return of the full range of tendon excursion [1].

Before the mid-1970s, most of the flexor tendon rehabilitation programs focused on late mobilization after the first 3 weeks following tendon repair as tendon tensile strength was considered low to perform active exercise during this period of time, but this ended in a high rate of tendon adhesions and limitation of range of movement [2].

Chow et al. in 1987 stated that only $3-5 \mathrm{~mm}$ tendon excursion was sufficient to prevent adhesion formation postoperatively [3]. Moreover, Edinburg et al. in 1987 showed good results of tendon function following immediate postoperative active extension passive flexion with attached rubber band and dorsal blocking splint [1]. Since this time, there is an accumulated expanding knowledge regarding suture

This is an open access journal, and articles are distributed under the terms of the Creative Commons Attribution-NonCommercial-ShareAlike 4.0 License, which allows others to remix, tweak, and build upon the work non-commercially, as long as appropriate credit is given and the new creations are licensed under the identical terms. 
materials, methods of tendon repair, and rehabilitation programs, which evolves toward early mobilization [4].

As it become evident that early passive rehabilitation programs are superior to prolonged immobilization regarding tendon excursion and gliding [5,6], several studies began investigating early active rehabilitation programs and found them more beneficial regarding tendon gliding and excursion $[6,7]$.

There is a gap in knowledge regarding the most appropriate rehabilitation program following flexor tendon injury because of sparse number of head-tohead comparison between early active rehabilitation programs protocols and early passive rehabilitation programs. To our knowledge, only Trumble et al. [8] discussed this point and stated that 'Active motion therapy provides greater active finger motion than passive motion therapy after zone II flexor tendon repair without increasing the risk of tendon rupture.' Yet this study was limited to four-strand repair and did not assess two-strand repair.

In our study, we randomized patients to an early active (place and hold) rehabilitation protocol compared with an early passive rehabilitation protocol (modified Kleinert) following two-strand repair of flexor tendon injuries, with validated outcome assessments of motion and patient satisfaction.

\section{Patients and methods \\ Patients}

This study was conducted in Physical Medicine and Rehabilitation Department, El Demerdash Hospital, Ain Shams University after approval of Ethical committee, and performing patient consent, over a period of 12 months between August 2015 and August 2016. It included 33 patients with 45 injured flexor tendons who presented to the emergency unit of the corresponding hospital fulfilling the following criteria:

(1) All flexor tendon injuries at all zones of the hand.

(2) Patients between the age of 15 and 75 years.

\section{Exclusion criteria}

The following were the exclusion criteria:

(1) Patients younger than 15 years, because of higher incidence of tendon rupture [9].

(2) Patients who are older than 75 years, as they have been shown to have deterioration of hand function scores, and normative data for these patients are not available [10].
(3) Patients with crush injury with extensive softtissue loss.

(4) Documented compliance problems (e.g. substance abuse).

(5) Those with medical conditions preventing repair and pre-existing problems such as arthritis limiting joint motion were also excluded.

A total of 33 patients (45 injured tendons) were enrolled in the study, and only 26 patients (36 tendons) continued the study, as seven patients were lost to follow-up. Overall, 20 (55.5\%) tendons were on early passive protocol (modified Kleinert) and 16 (44.4\%) tendons were on the early active protocol (place and hold).

The patients comprised 21 (80.7\%) males and five (19.2\%) females; their age ranged from 15 to 60 years, with a mean of 26.8 years. Regarding the patients' occupation, $15(57.67 \%)$ patients were manual workers, four (15.3\%) patients were housewives, four (15.3\%) patients were students, and three (11.5\%) patients had professional jobs (Table 1).

Of the patients' injuries, seven (19.4\%) tendon injuries occurred in zone I, 22 (61.1\%) tendon injuries occurred in zone II, seven (19.4\%) tendon injuries in zone III, and $0 \%$ in zone IV and zone V. There were eight $(22.58 \%)$ patients with more than one tendon affection. There were 11 (35.48\%) fingers that had associated neurovascular injuries.

The distribution of the injured fingers was as follow: six (16.6\%) tendons were flexor pollicis longus, 13 (36.1\%) tendons were index finger, eight (22.2\%) tendons were middle finger, three (8.3\%) tendons were ring finger, and six (16.6\%) tendons were little finger. Of the injured fingers $47.2 \%$ (17 tendons) occurred in the nondominant hand, and $52.7 \%$ (19 tendons) occurred in the dominant hand (Table 2)

\section{Surgical technique}

All tendons were repaired by experienced hand surgeons who had at least between 4 and 6 years of training in hand

Table 1 Demographic data

\begin{tabular}{lc}
\hline Categories & $n(\%)$ \\
\hline Sex & \\
$\quad$ Male & $21(80.7)$ \\
Female & $5(19.2)$ \\
Occupation & \\
Manual worker & $15(57.67)$ \\
Housewife & $4(15.3)$ \\
Student & $4(15.3)$ \\
Professional job & $3(11.5)$ \\
\hline
\end{tabular}




\begin{tabular}{lc}
\hline & $n(\%)$ \\
\hline Dominant hand & $13(50)$ \\
Nondominant hand & $13(50)$ \\
Affected finger & \\
Thumb & $6(16.6)$ \\
Index finger & $13(36.1)$ \\
Middle finger & $8(22.2)$ \\
Ring finger & $3(8.3)$ \\
Little finger & $6(16.6)$ \\
Associated injuries & \\
Associated nerve injury & $7(22.58)$ \\
Associated arterial injury & $7(22.58)$ \\
Associated fracture & $3(9.7)$ \\
Zones & \\
1 & $7(19.4)$ \\
2 & $22(61.1)$ \\
3 & $7(19.4)$ \\
4 & $0(0)$ \\
5 & $0(0)$ \\
\hline
\end{tabular}

surgery. The wound was extended using Bruner incisions, and a flap was raised to expose the tendon, preserving the functionally important $\mathrm{A} 2$ and $\mathrm{A} 4$ pulleys. Pull out suture was made for zone 1 injury with short distal stump $(<1 \mathrm{~cm})$. The used suture materials were $3 / 0$ or $4 / 0$ prolene for core suture modified Kessler technique and $5 / 0$ or $6 / 0$ prolene for epitendinous sutures. Associated digital nerve and arterial injuries were repaired by the $8 / 0$ or $9 / 0$ ethibond, and the tendon rehabilitation protocol was not altered.

\section{Postoperative rehabilitation}

All the patients were splinted in dorsal blocking splint with wrist in $20^{\circ}$ flexion, Metacarpo-phalangeal joint (MCP) joint in $70^{\circ}$ flexion and interphalangeal joints in full extension. The splint was removed after 6 weeks. We randomized patients into two groups by random sequence-generating website [11]: both groups started rehabilitation program within the first 3 days after tendon repair under the supervision of a hand rehabilitation consultant.

One group underwent early passive rehabilitation program by modified Kleinert method (passive flexion and active extension) in addition to passive range of motion for all joints.

The other group received early active rehabilitation programs (place and hold) wherein the digit is passively flexed by the unaffected hand and then the patient tries to maintain the flexed posture through active contraction of the involved muscle for $5 \mathrm{~s}$ [12], in addition to passive range of motion for all joints, and passive flexion active extension, progressed to active tenodesis. Each patient was instructed to do these exercises 25 times per wakening hours for the first 6 weeks.

Then assessment began at sixth week postoperatively measuring the following outcomes:

(1) Flexion deformity and tendon lag: by using goniometer, this will be done by measurement of degree of flexion deformity in proximal interphalangeal (PIP) and distal interphalangeal (DIP). Then measuring the difference between active and passive range of motion for both joints to assess flexor digitorum superficialis (FDS) and flexor digitorum profundus (FDP).

(2) Disability assessment by validated Disabilities of the Arm, Shoulder and Hand (DASH) questionnaire was done at 6 months from the time of surgery to compare outcomes [13].

(3) Patients' satisfaction with their hand function was measured on an analog scale from 0 (completely dissatisfied) to 10 (completely satisfied).

(4) Postoperative complications including the following:

(a) Rupture rate.

(b) Adherent scar formation.

\section{Data management and analysis}

The collected data were revised, coded, tabulated, and introduced to a PC using statistical package for the social science (SPSS 15.0.1 for Windows, 2001; SPSS Inc., Chicago, Illinois, USA). Data were presented, and suitable analysis was done according to the type of data obtained for each parameter.

\section{Descriptive statistics}

Mean $\pm S D$ and range for parametric numerical data whereas median and interquartile range (IQR) for nonparametric numerical data were used. Frequency and percentage were used for non-numerical data.

\section{Analytical statistics}

Mann-Whitney test ( $U$ test) was used to assess the statistical significance of the difference of a nonparametric variable between the two study groups. Student's $t$ test was used to assess the statistical significance of the difference between the two study groups. The $P$ value was considered significant if less than $0.05 \%$. No data were available on DASH to assist with the power analysis. The primary outcomes of this study were range of motion and rupture rate. The secondary outcomes were scar formation, patient satisfaction assessed by DASH score and analog score from 0 to 10 (Diagram 1). 


\section{CONSORT Flow Diagram}

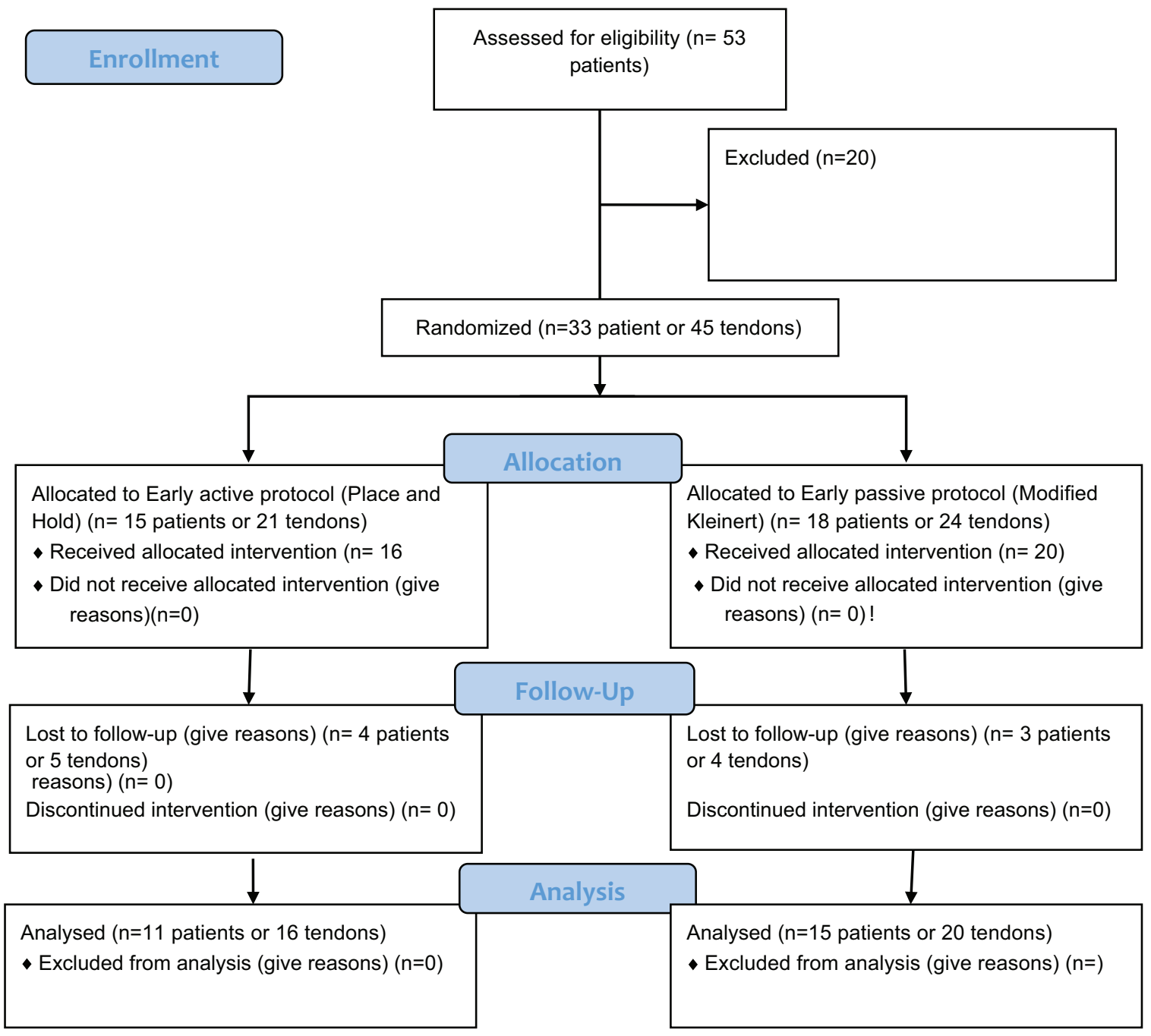

CONSORT flow diagram.

\section{Results}

Of the 33 enrolled patients (45 injured tendons), only 26 patients (36 tendons) continued the study, as seven patients were lost to follow-up. A total of 20 (55.5\%) tendons were on early passive protocol (modified Kleinert) and 16 (44.4\%) tendons were on the early active protocol (place and hold). The patients comprised 25 (80.6\%) males and six (19.3\%) females, and their age ranged from 15 to 60 years, with a mean of 26.8 years.

Overall, 10 (50\%) tendons of the 20 tendons underwent passive program showed flexion deformity in DIP, whereas in the active program, one (6.2\%) of 16 tendons showed DIP flexion deformity. There was a statistically significant difference between the two groups $(P=0.004)$. Regarding the flexion deformity in the PIP joint, $40 \%$ (eight tendons) of the passive group had a degree of flexion deformity and $18.7 \%$ (three tendons) of the active group also complained of a degree of flexion deformity, with no statistically significant difference between the two groups $(P=0.1)$. However, combined flexion deformities of both DIP and PIP joints showed significant difference between active and passive groups $(P=0.02)$ (Fig. 1).

There was a degree of tendon lag in FDP and FDS in both groups. The FDS tendon lag in passive group was 50\% (10 tendons) and 12.5\% (two tendons) in active group, with statistically significant difference $(P=0.012)$. The FDP tendon lag also showed 
statistically significant difference between the two groups $(P<0.001)$ with $75 \%$ (15 tendon) in passive group and $12.5 \%$ (two tendons) in active group, and also for combined tendon lag for both tendons, there was a significant difference $(P<0.001)$ (Fig. 2$)$.

The patient satisfaction with their hand function was measured on an analog scale from 0 (completely

Figure 1

\section{Rupture rate}

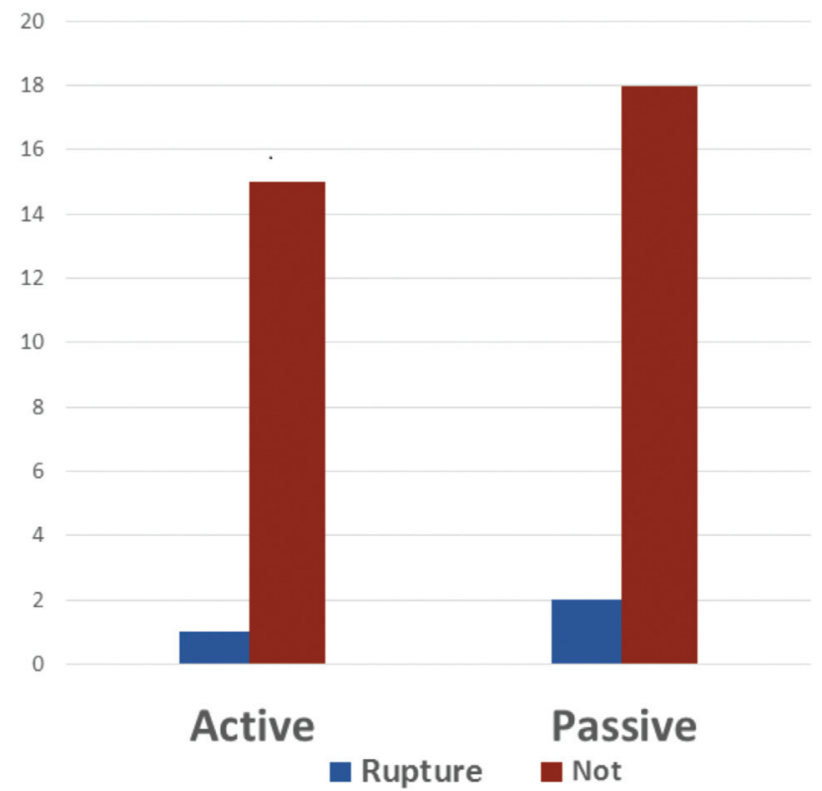

Flexion deformity in both distal interphalangeal (DIP) joints and proximal interphalangeal (PIP) joints. dissatisfied) to 10 (completely satisfied). The median score for the patient satisfaction in the active group was 6 with IQR between 4 and 8.5, whereas in the passive group, it was 4 with IQR between 2 and 7 . These values showed a statistically significant difference between the two groups $(P=0.049)$.

DASH questionnaire, which was used for objectively assessing the hand function from the patient perspective, revealed that the passive group showed a median of 15 with IQR between 10-and 30, whereas the active group showed a median of 23 with IQR between 2 and 26 . These results show no statistically significant difference $(P=0.62)$.

Regarding the adherent scar formation, there was a statistically significant difference between the two groups ( $P=0.001$ ) with $70 \%$ (14 tendons) in the passive group and $0 \%$ (0 tendons) in the active group (Fig. 3).

Regarding the rupture rate, the passive group showed two (10\%) rupture tendons of the 20 tendons, whereas the active group has one $(6.25 \%)$ rupture tendon of the 16 tendons, with no statistically significant difference between the two groups (Fig. 4).

There were 11 patients with associated repaired digital nerve and vessels, four in the active rehabilitation group and seven in the passive group, who continued on the
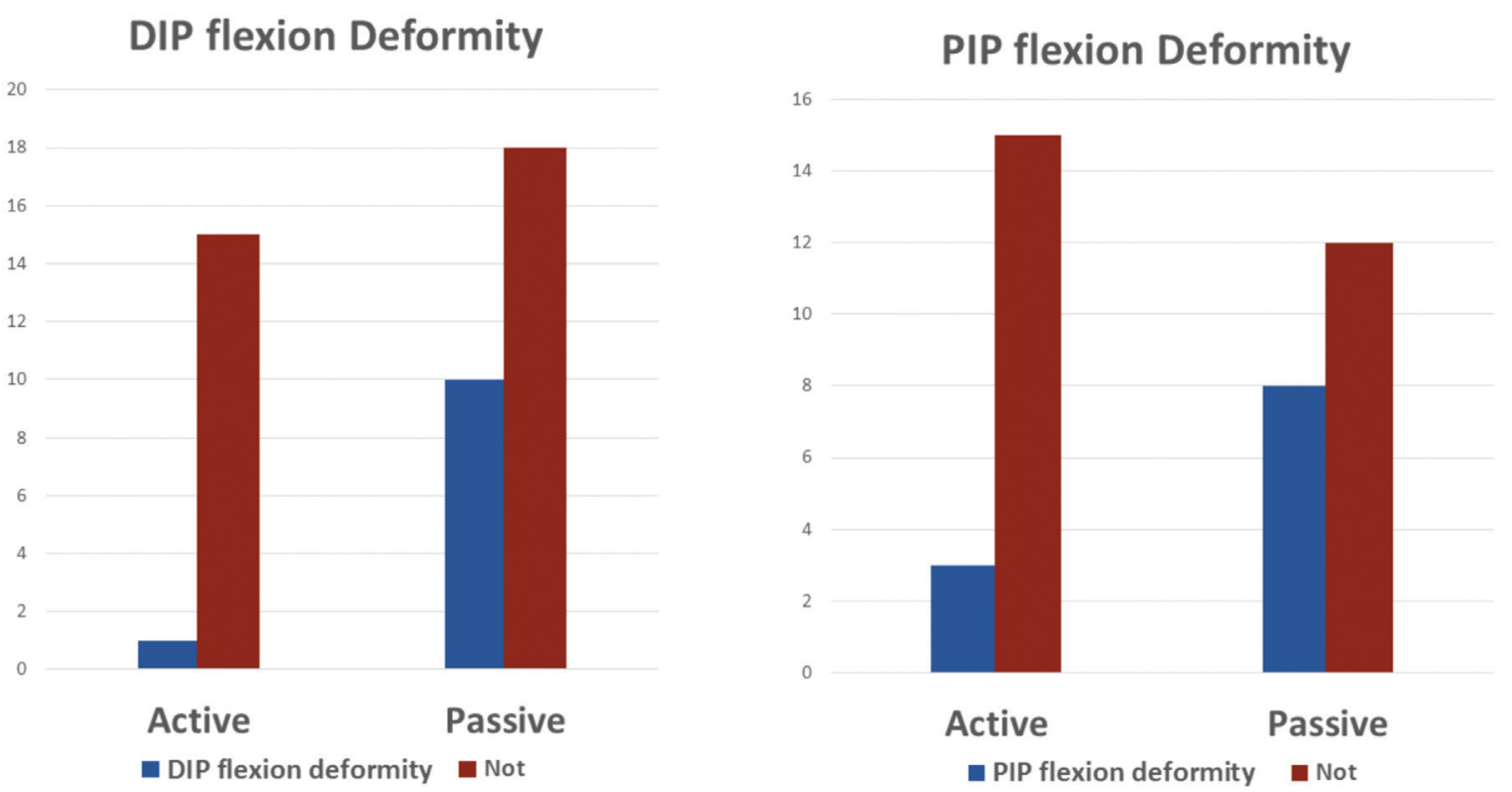

Flexor digitorum superficialis (FDS) and flexor digitorum profundus (FDP) tendon lag. 

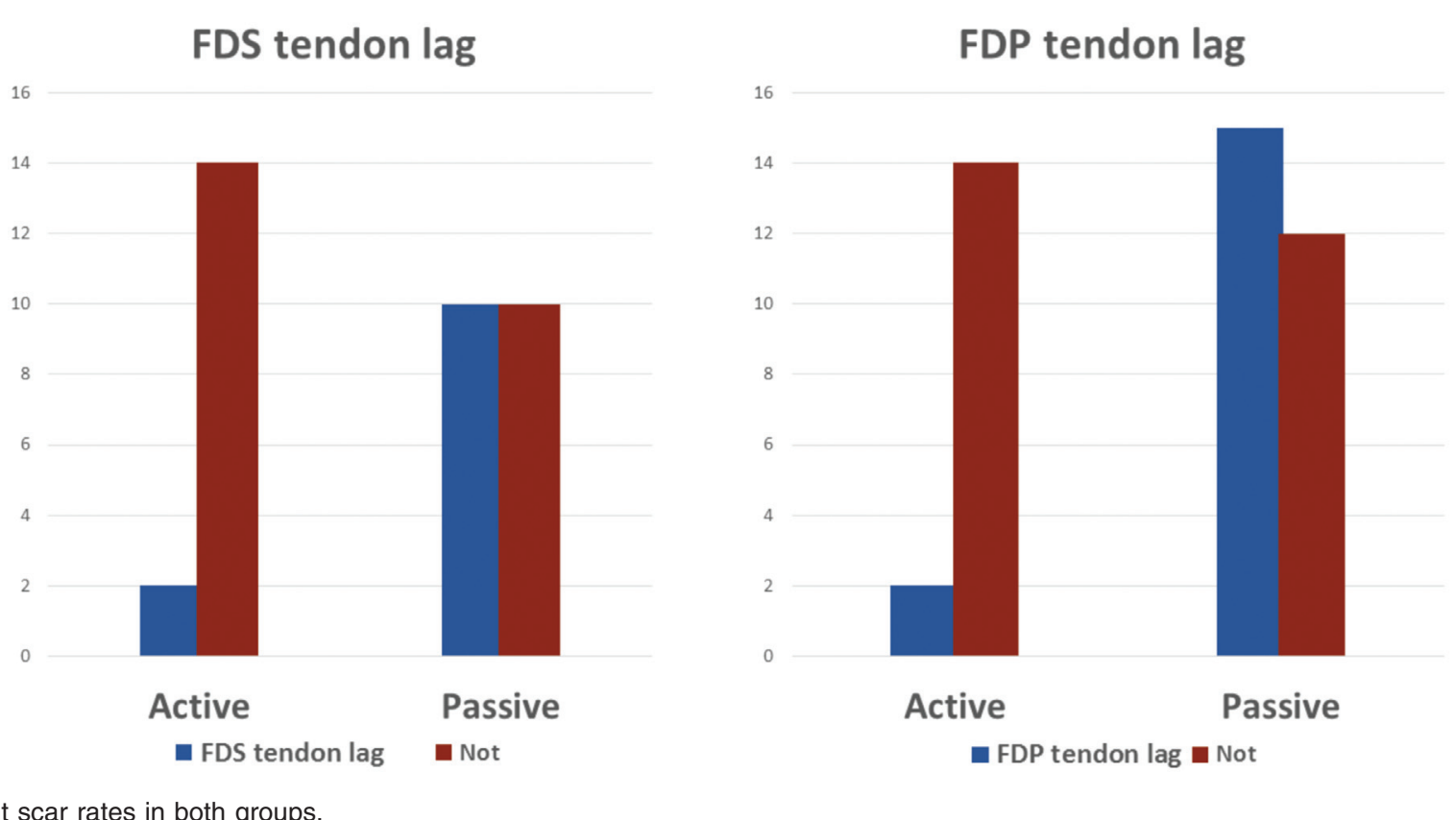

\section{Figure 4}

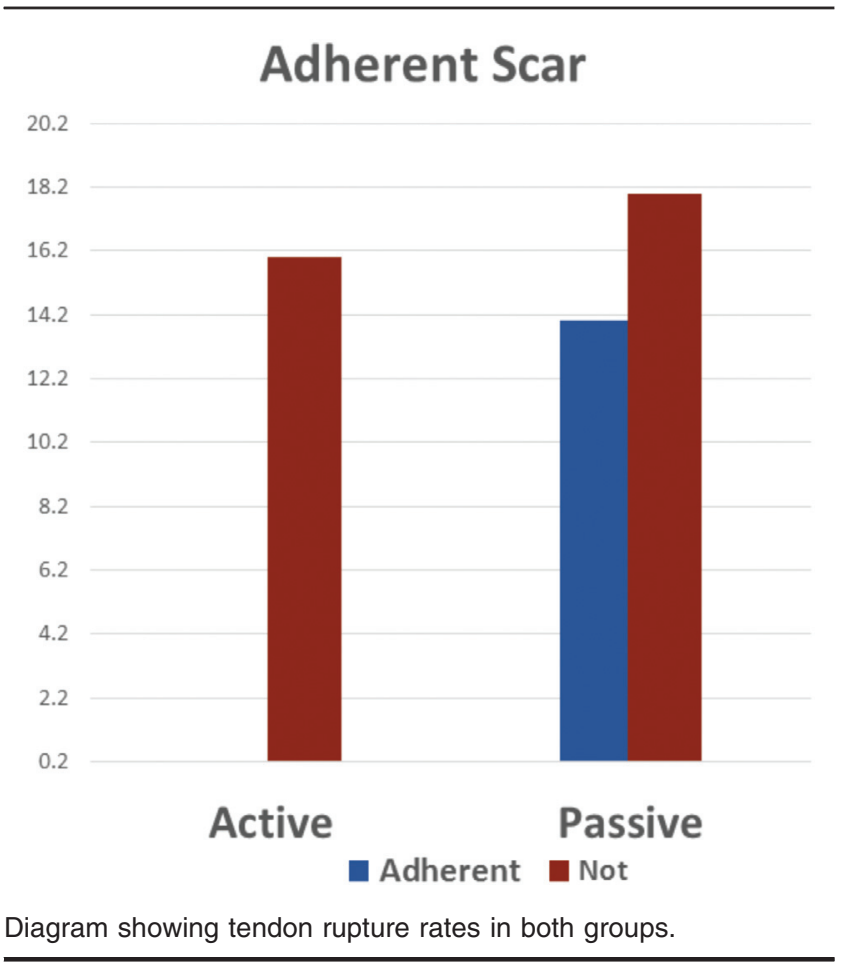

same program with no incidence of rupture in any of them.

\section{Discussion}

Full return to the active digital range of motion as well as prevention of joint contraction and adhesions following flexor tendon repair is a challenging problem facing hand surgeons worldwide. Many modalities of rehabilitation program were advocated since 1960 [14]. The immobilization program was the first program to be considered. Then, because of the poor results of the previously advocated immobilization programs [1], Kleinert and colleagues [15-18] developed controlled motion protocol based on active extension passive flexion of the injured digit within a dorsal blocking splint.

As it became evident that early passive mobilization has a superior outcomes over immobilization [18-20], several authors began to think about controlled early active mobilization. Their studies yielded that early active mobilization has better tendon gliding with fewer adhesions [21,22]. Yet, the risk tendon rupture was still one of the obstacles against early active mobilization protocols [23].

There is a great gap of knowledge in the literature about the most appropriate rehabilitation programs after flexor tendon injury. This is owing to the sparse number of randomized control trails comparing different rehabilitation protocols. On the contrary, this puts a great burden upon the hand surgeon when choosing studies advocating a specific technique, with no controls. Even the few controlled studies conducted vary in methods of repair, splinting positions, start time of rehabilitation, and outcome assessment [19,24].

Silfverskiöld and May [7] demonstrated that the tendon excursion and gliding was better in the active 
extension passive flexion group compared with the only passive motion protocol. Trumble et al. [8], after performing a randomized study comparing active versus passive programs following standard fourstrand repair of flexor tendon injuries in zone II, stated that patients on active rehabilitation program had better range of motion with less flexion contractures and greater satisfaction scores than those in the passive rehabilitation protocol.

In our study, we postulated that early active mobilization will lead to better tendon gliding and excursion even with the two-strand repair as active motion will decrease adhesion formation.

Comparing our findings with the results of Trumble et al. [8], who found that the rupture rate in active group was $4.4 \%$ (two of 45 tendons) and the rupture rate in passive group was $4.5 \%$ (two of 44 tendons). Of these four ruptures, three occurred in the little finger. In our study, we have one rupture case in the active group of the $16(6.25 \%)$ tendons and two (10\%) rupture cases in the passive group of 20 tendons, with no statistically significant difference $(P=0.6)$, denoting that the active program with two-strand repair can withstand early mobilization with place-and-hold technique.

Regarding the tendon lag and flexion deformity, Trumble et al. [8] found that at 6 weeks there is a statistically significant difference between active and passive groups. The combined flexion contracture of the PIP and DIP joints for active group averaged $27 \pm 12$ at 6 weeks, and the flexion contracture in the passive group averaged $42 \pm 19$ at the same time [8].

In our study, we found that the combined flexion deformity of DIP and PIP in active group at 6 weeks averaged $3.1 \pm 6.3$ and for the passive group averaged $27.8 \pm 43.8$, with $P$ value of 0.02 , denoting a statistically significant difference, which occurs owing to the tenodesis mobilization that is performed in the early active mobilization group, as during wrist flexion, the tension on extensor tendons brings the digits into extension, whereas during extension of the wrist, tension in flexor tendons brings digits into flexion. This mobilization helps to reduce edema and joint stiffness and promote proximal gliding of flexor tendons.

However, for the tendon lag, there is significant difference between active and passive groups for combined tendon lag for both FDS and FDP $(P<0.001)$, and that is owing to active mobilization with maximal extension of the interphalangeal joints that promote passive gliding of the flexor tendons [22]. When performed carefully, this can give rise to good clinical results, although there is a risk of rupturing the repair [23]. Moreover, active flexion of the finger contributes toward differential gliding between the flexor tendons. Moreover, active muscle contraction promotes recovery of muscle tone and strength. The loading that is applied to the repaired tendon during active finger flexion improves the tensile strength after healing [24].

On the contrary, the DASH score at 6 months postoperatively for the active group averaged 19.1 \pm 18.9 , whereas for the passive group averaged 20.2 \pm 14.4 , with no statistically significant difference $(P=0.84)$. In the study by Trumble et al. [8], DASH score at 1-year postoperatively showed significant difference between the two groups $(P=0.09)$, as the active group averaged $2 \pm 3.7$, whereas the passive group averaged $3.1 \pm 4.3$.

Regarding adherent scar formation, there was a statistically significant difference between healing processes. Moreover, a difference in the beneficial effect of increased blood flow and increased oxygenation was seen in the two groups $(P=0.001)$, with $70 \%$ (14 tendons) in the passive group and $0 \%(0$ tendons) in the active group.

The median of the patient satisfaction score in the passive group was 4 with IQR of $4-8.5$, whereas in the active group was 6.00 with IQR of 4-8.5. There was a statistically significant difference between the two groups $(P=0.049)$.

Previously, Charles et al. [25] showed that active exercises has a beneficial effect on wound healing, via neuroendocrine regulation and changes in cortisol responsivity, which result in immune function changes relevant to the wound, thereby enhancing wound-healing rates.

All of these agree with the study of Mikkawy et al. [26], which showed that early active mobilization in flexor tendon has a higher mean average grip strength and earlier improvement than the passive mobilization program.

The limitations in our study was the number of patients that continued the total follow-up which were for 6 months, so we lost a lot of patients. This is attributed to the economic burden of the follow-up period, as most of the patients are manual workers so they could not complete their regular visits. 


\section{Conclusion}

Early active mobilization had a better tendon gliding and excursion even with the two-strand repair, as active motion will decrease adhesion formation, with significant difference to the passive group. Moreover, there was no significant difference in the rupture rate and significant difference for combined tendon lag and flexion deformity owing to the tenodesis mobilization between both the groups.

\section{Financial support and sponsorship}

Nil.

\section{Conflicts of interest}

There are conflicts of interest.

\section{References}

1 Edinburg M, Widgerow AD, Biddulph SL. Early postoperative mobilization of flexor tendon injuries using a modification of the Kleinert technique. J Hand Surg 1987; 12:34-38.

2 Strickland JW, Glogovac SV. Digital function following flexor tendon repair in Zone II: a comparison of immobilization and controlled passive motion techniques. J Hand Surg Am 1980; 5:537-543.

3 Chow JA, Thomes LJ, Dovelle S, Milnor WH, Seyfer AE, Smith AC. A combined regimen of controlled motion following flexor tendon repair in 'no man's land. Plast Reconstr Surg 1987; 79:447-455.

4 Bunker TD, Potter B, Barton NJ. Continuous passive motion following flexor tendon repair. J Hand Surg $\mathrm{Br}$ 1989; 14:406-411.

5 Osada D, Fujita S, Tamai K, Yamaguchi T, Iwamoto A, Saotome K. Flexor tendon repair in zone II with 6-strand techniques and early active mobilization. J Hand Surg Am 2006; 31:987-992.

6 Starr HM, Snoddy M, Hammond KE, Seiler JG. Flexor tendon repair rehabilitation protocols: a systematic review. J Hand Surg Am 2013; 38:1712-1717.

7 Silfverskiöld KL, May EJ. Flexor tendon repair in zone II with a new suture technique and an early mobilization program combining passive and active flexion. J Hand Surg Am 1994; 19:53-60.

8 Trumble TE, Vedder NB, Seiler JG, Hanel DP, Diao E, Pettrone S. Zone-II flexor tendon repair: a randomized prospective trial of active place-and-hold therapy compared with passive motion therapy. J Bone Joint Surg Am 2010; 92:1381-1389.
9 Fitoussi F, Lebellec Y, Frajman JM, Pennecot GF. Flexor tendon injuries in children: factors influencing prognosis. J Pediatr Orthop 1999; 19:818-821.

10 Jebsen RH, Taylor N, Trieschmann RB, Trotter MJ, Howard LA. An objective and standardized test of hand function. Arch Phys Med Rehabil 1969; 50:311-319.

11 Urbaniak GC, Plous S. Research randomizer (version 4.0) (computer software).2013. Available at: http://www.randomizer.org/. [Accessed 22 June 2013].

12 Pettengill KM. The evolution of early mobilization of the repaired flexor tendon. J Hand Ther 2005; 18:157-168.

13 Hudak PL, Amadio PC, Bombardier C. Development of an upper extremity outcome measure: the DASH (disabilities of the arm, shoulder and hand) [corrected]. The Upper Extremity Collaborative Group (UECG). Am J Ind Med 1996; 29:602-608.

14 Chow JA, Thomes LJ, Dovelle S, Milnor WH, Seyfer AE, Smith AC. A combined regimen of controlled motion following flexor tendon repair in' no man's land'. Plastic Reconstr Surg 1987; 79:447-453.

15 Lister GD, Kleinert HE, Kutz JE, Atasoy E. Primary flexor tendon repair followed by immediate controlled mobilization. J Hand Surg Am 1977; 2:441-451.

16 Kleinert HE, Spokevicius S, Papas NH. History of flexor tendon repair. J Hand Surg Am 1995; 20:S46-S52.

17 Kleinert HE, Lubahn JD. Current state of flexor tendon surgery. Ann Chir Main 1984; 3:7-17.

18 Kleinert HE, Kutz JE, Atasoy E, Stormo A. Primary repair of flexor tendons. Orthop Clin North Am 1973; 4:865-876.

19 Strickland JW. Flexor tendon repair. Hand Clin 1985; 1:55-68.

20 Nielsen AB, Jensen PO. Primary flexor tendon repair in 'no man's land. J Hand Surg Br 1984; 9:279-281.

21 Becker H, Orak F, Duponselle E. Early active motion following a beveled technique of flexor tendon repair: report on fifty cases. J Hand Surg Am 1979; 4:454-460.

22 Small JO, Brennen MD, Colville J. Early active mobilisation following flexor tendon repair in zone 2. J Hand Surg Br 1989; 14:383-391.

23 Peck FH, Bücher CA, Watson JS, Roe A. A comparative study of two methods of controlled mobilization of flexor tendon repairs in zone 2. J Hand Surg Br 1998; 23:41-45.

24 Chow JA, Thomes LJ, Dovelle S, Monsivais J, Milnor WH, Jackson JP. Controlled motion rehabilitation after flexor tendon repair and grafting. A multi-centre study. Bone Jt J 1988; 70:591-595.

25 Charles F, Emery JK, Kiecolt-Glaser R, Glaser WB, Malarkey J. Exercise accelerates wound healing among healthy older adults: a preliminary investigation. J Gerontol 2005; 60:1432-1436.

26 Mikkawy D, Amr A, Gad A, Lasheen R, Fawaz S, Abd Elsabour H. Comparison between early active and passive mobilization programs after hand flexor tendon repair in zone II. Egypt Rheumatol Rehab J 2013; 40:134-140. 